\title{
Structural evolution during protein denaturation as induced by different methods
}

\author{
S. Chodankar, ${ }^{1}$ V. K. Aswal, ${ }^{1}$ J. Kohlbrecher, ${ }^{2}$ R. Vavrin, ${ }^{2}$ and A. G. $\mathrm{Wagh}^{1}$ \\ ${ }^{1}$ Solid State Physics Division, Bhabha Atomic Research Centre, Mumbai-400 085, India \\ ${ }^{2}$ Laboratory for Neutron Scattering, ETH Zurich \& Paul Scherrer Institut, CH-5232 Villigen PSI, Switzerland
}

(Received 22 October 2007; revised manuscript received 19 November 2007; published 4 March 2008)

\begin{abstract}
Small-angle neutron scattering (SANS) and dynamic light scattering (DLS) have been used to study conformational changes in protein bovine serum albumin (BSA) due to perturbation in its native structure as induced by varying temperature and pressure, and in presence of protein denaturating agents urea and surfactant. BSA has prolate ellipsoidal shape at ambient temperature and we observe no effect of temperature on its structure up to a temperature of about $60^{\circ} \mathrm{C}$. At temperatures beyond $60{ }^{\circ} \mathrm{C}$, protein denaturation leads to aggregation. The protein solution exhibits a fractal structure at temperatures above $64{ }^{\circ} \mathrm{C}$, and its fractal dimension increases with temperature. This is an indication of aggregation followed by gelation that evolves with increasing temperature. It is known for some of the proteins (e.g., Staphylococcal Nuclease) that pressure of $200 \mathrm{MPa}$ can unfold the protein, whereas BSA does not show any protein unfolding even up to the pressure of $450 \mathrm{MPa}$. In presence of urea, the BSA protein unfolds for urea concentrations greater than $4 M$ and acquires a random coil configuration. We make use of the dilution method to show the reversibility of protein unfolding with urea. The addition of surfactant denaturates the protein by the formation of micellelike aggregates of surfactants along the unfolded polypeptide chains of the protein. We show such structure of the proteinsurfactant complex can be stabilized at higher temperatures, which is not the case for pure protein.
\end{abstract}

DOI: 10.1103/PhysRevE.77.031901

PACS number(s): 87.14.E-, 87.15.N-, 61.05.fg

\section{INTRODUCTION}

Protein denaturation is one of the most widely studied topics in molecular biology due to its wide spread application in the industrial and scientific world. The denaturation process can be brought about by various means and conditions [1-5]. Each different route of denaturation has its own application and advantage in material processing and basic sciences. For instance, the temperature and pressure induced denaturation as used in food processing industry [6-8], whereas the surfactant induced denaturation is known to play an important role in the pharmaceutical and cosmetic industry $[9,10]$. Urea is being used for long time to understand the fundamental process of protein folding and unfolding [4,11]. Along with different applications related with different denaturation processes, these methods have different mechanism of denaturating the proteins. For example, it is believed that a temperature rise results in weakening and subsequently breaking of bonds in the protein macromolecule. As these bonds are broken, hydrophobic groups hidden in the protein core are exposed to the solvent $[12,13]$. On the other hand, pressure unlike temperature results in transfer of solvent to the hydrophobic core of the protein. The weakening of the hydrophobic interactions between the nonpolar side chains due to penetration of solvent results in the pressure induced denaturation $[14,15]$. The addition of denaturating agents such as urea and surfactant also leads to protein denaturation, following a different route than that in the case of temperature and pressure. In presence of urea, both the direct and indirect mechanisms have been discussed in the literature for the understanding of protein denaturation [16-23]. The direct mechanism involves urea $\mathrm{H}$ bonding to the peptide backbone, thereby favoring the denaturated state [16-19]. In indirect mechanisms some of the strong $\mathrm{H}$ bonds between water molecules are broken in the presence of urea, which results in solvation of hydrophobic groups [20-23]. Despite recent indications in favor of direct mechanisms [16-19], protein denaturation via both the direct and indirect mechanism has been emphasized as well [23]. Protein denaturation in the case of amphiphilic molecules such as surfactant is caused by the binding of these molecules to the hydrophobic patches of the protein $[24,25]$.

The function of a protein depends absolutely on its threedimensional folded structure [2]. A denaturation process involves the disruption of $\mathrm{H}$ bonds, disulphide bonds, salt bridges, and hydrophobic interactions, leading to the successive alteration of quaternary, tertiary, and secondary structure. However, peptide bonds are not broken leaving the primary structure unaltered. Different environmental parameters responsible for protein denaturation compel it to acquire a certain structure. Various techniques are being used to understand the structure of a protein and the mechanism of its changes during denaturation under different conditions [26-29]. Earlier studies have proposed that temperatureinduced denaturation leads to the formation of a threedimensional continuous network of protein aggregates, i.e., a gel phase. This gel phase can be characterized by its fractal nature $[30,31]$. In contrast, pressure induced denaturation studies have shown that an application of pressure leads individual protein to unfold to a random coil polymer [32]. Similar to the pressure effect, the addition of urea is known to unfold a protein into a polypeptide chain which acquires a random coil conformation $[4,11]$. In the presence of surfactant, the binding of surfactant on protein results in micellelike aggregates enclosing the hydrophobic patches on the protein backbone. This leads to acquisition of a necklacebead structure of the protein surfactant complex [33-35].

Protein denaturation has been studied using various methods such as viscometry [36], circular dichroism [37,38], nuclear magnetic resonance (NMR) [39,40], and scattering techniques $[41,42]$. These methods probe denaturation with 
different resolutions. For example, viscometry explains the denaturation of proteins based on the increase in viscosity, due to the entanglement of unfolded protein [36]. Circular dichroism gives information about the changes in the helical content in the secondary structure, but does not provide information about the overall changes in the three-dimensional structure of the protein [37]. NMR indicates unfolding from the chemical shift, which is due to the difference in interactions of the folded and unfolded parts of the protein with the solvent [40]. Scattering techniques correlate the denaturation with the conformational changes in the three-dimensional structure of the protein $[41,42]$. In the present paper, we have used small-angle neutron scattering (SANS) and dynamic light scattering (DLS) to probe conformational changes during the protein denaturation induced by four different methods. The experiments are performed on bovine serum albumin (BSA) protein, which is one of the commonly used proteins for various studies. The structural evolution of the denaturating protein has been studied at different temperatures $\left(60-74{ }^{\circ} \mathrm{C}\right)$. The effect of pressure on the protein is examined up to $450 \mathrm{MPa}$. Protein denaturation in presence of urea is studied over a wide concentration range of urea $(0 M-8 M)$ and reversibility of denaturation has been evaluated by the dilution method. The temperature effect on surfactant induced denaturation has been studied for different surfactant concentrations $[0 M-40 \mathrm{~m} M]$. SANS with the possibility to vary the contrast is an ideal technique for studying hydrogenous systems such as protein solution. This technique provides information about the geometry and conformation of the scattering particles [43]. DLS is a complimentary technique to SANS, which gives size information by measuring the diffusion coefficient of the particle [27].

\section{EXPERIMENT}

BSA protein (catalog No. 05480), SDS surfactant (catalog No. 71727) and urea were purchased from Fluka. Samples for SANS experiments were prepared by dissolving known amount of BSA and other additives (surfactant or urea) in a buffer solution of $\mathrm{D}_{2} \mathrm{O}$. The use of $\mathrm{D}_{2} \mathrm{O}$ as solvent instead of $\mathrm{H}_{2} \mathrm{O}$ provides better contrast for hydrogenous protein in neutron experiments. The interparticle interactions in these systems were minimized by preparing the samples in acetate buffer solution at $\mathrm{pH} 5.4$, which is close to the isoelectric $\mathrm{pH}$ of BSA (4.9), and at high ionic strength of $0.5 \mathrm{M} \mathrm{NaCl}$. Small-angle neutron scattering experiments were performed on the SANS-I instrument at Swiss Spallation Neutron Source, SINQ, Paul Scherrer Institut, Switzerland [44]. The mean wavelength of the incident neutron beam was $6 \AA$ with a wavelength resolution of approximately $10 \%$. The scattered neutrons were detected using two-dimensional $96 \mathrm{~cm}$ $\times 96 \mathrm{~cm}$ detector. The experiments were performed at two sample-to-detector distances of 2 and $8 \mathrm{~m}$, respectively to cover the data in the wave vector transfer $\mathrm{Q}$ range of 0.006 to $0.25 \AA^{-1}$. The measured SANS data were corrected and normalized to a cross-sectional unit using BERSANS-PC data processing software [45]. DLS measurements on above samples were carried out using a commercial ALV/LSE-5003 light scattering instrument featuring a multiple tau digital correlator. The light source was a helium neon laser operated at $6328 \AA$. All the measurements were performed at a scattering angle of $90^{\circ}$ and the instrument has an angular resolution of $0.05^{\circ}$. The temperature was kept fixed at $30{ }^{\circ} \mathrm{C}$ for all the measurements except for temperature dependent study in which temperature was varied in the range $30-74{ }^{\circ} \mathrm{C}$. In the analysis, refractive index of water has been used for all the DLS measurements. The changes in the refractive index with increasing temperature or adding urea have been used with those reported in the literature [46,47]. Pressure dependence measurements on protein solutions were performed using a $500 \mathrm{MPa}$ high-pressure cell having two parallel thick sapphire windows. For DLS measurements under high pressure a separate in-house built setup was used as the commercial ALV setup cannot mount the high-pressure cell [48]. The scattering is measured at an angle of $135^{\circ}$, which is calibrated by comparing the results of the standard polymer samples measured on this instrument with that of ALV instrument. For both the above DLS setups, measurements have been performed in homodyne configuration.

\section{DATA ANALYSIS}

\section{A. Small-angle neutron scattering}

In small-angle neutron scattering one measures the coherent differential scattering cross-section per unit volume $[d \Sigma / d \Omega(Q)]$ as a function of $Q$. For a system of monodispersed interacting protein macromolecules, $d \Sigma / d \Omega(Q)$ can be expressed as [49]

$$
\frac{d \Sigma}{d \Omega}(Q)=N_{p} V_{p}^{2}\left(\rho_{p}-\rho_{s}\right)^{2}\left[\left\langle F(Q)^{2}\right\rangle+\langle F(Q)\rangle^{2}\left(S_{p}(Q)-1\right)\right]+B,
$$

where $N_{p}$ is the proteins number density and $V_{p}$ is the volume of the protein macromolecule. $\rho_{p}$ and $\rho_{s}$ are the scattering length density of the protein and the solvent, respectively. $F(Q)$ is the single particle form factor and $S_{p}(Q)$ is the interparticle structure factor. $B$ is a constant term that represents the incoherent scattering background, which is mainly due to hydrogen in the sample.

In general, charged colloidal systems such as protein solutions show a correlation peak in the SANS data [50]. The peak arises because of the interparticle structure factor $S_{p}(Q)$ and indicates the presence of significant interaction (electrostatic and/or hard sphere) between the colloids. In the case of a solution with low protein concentration, having high salt concentration and $\mathrm{pH}$ close to isoelectric point of the protein, $S_{p}(Q)$ can be approximated to unity as the interparticle interactions are minimized, and Eq. (1) for such system becomes

$$
\frac{d \Sigma}{d \Omega}(Q)=N_{p} V_{p}^{2}\left(\rho_{p}-\rho_{s}\right)^{2}\left\langle F(Q)^{2}\right\rangle+B
$$

The single particle form factor of the protein macromolecules in their native conformation has been calculated by treating them as prolate ellipsoids. For such an ellipsoidal particle [51] 


$$
\begin{aligned}
& \left\langle F^{2}(Q)\right\rangle=\int_{0}^{1}\left[F(Q, \mu)^{2} d \mu\right], \\
& F(Q, \mu)=\frac{3(\sin x-x \cos x)}{x^{3}}, \\
& x=Q\left[a^{2} \mu^{2}+b^{2}\left(1-\mu^{2}\right)\right]^{1 / 2},
\end{aligned}
$$

where $a$ and $b$ are, respectively, the semimajor and semiminor axes of the ellipsoidal protein macromolecules and $\mu$ is the cosine of the angle between the directions of $a$ and the wave vector transfer $Q$.

Temperature-induced denaturation of proteins leads to aggregation followed by gelation. For such a system the structure factor is not anymore negligible and has to be included in the description of the scattering cross section. The aggregation process starts with individual protein macromolecules which aggregate into a larger network formation. The cross section for such a system can be expressed as [52]

$$
\frac{d \Sigma}{d \Omega}(Q) \sim P_{p}(Q) S_{f}(Q)+B
$$

$P_{p}(Q)$ denotes the normalized intraparticle structure factor of a protein macromolecule in the cluster, which is the building block of the complex gel and can be considered to be an equivalent sphere of radius $R_{p} . P_{p}(Q)$ for a spherical particle of radius $R_{p}$ is given by

$$
P_{p}(Q)=\left[\frac{3\left(\sin Q R_{p}-Q R_{p} \cos Q R_{p}\right)}{\left(Q R_{p}\right)^{3}}\right]^{2} .
$$

$S_{f}(Q)$ has been calculated using fractal structure of the aggregated network. In this case, $S_{f}(Q)$ is given as [53]

$$
\begin{aligned}
S_{f}(Q)= & 1+\frac{1}{\left(Q R_{p}\right)^{D}} \frac{D \Gamma(D-1)}{\left.\left[1+(Q \xi)^{-2}\right]^{[(D-1) / 2}\right]} \\
& \times \sin \left[(D-1) \tan ^{-1}(Q \xi)\right],
\end{aligned}
$$

where $D$ the fractal dimension of the gel and $\xi$ is the correlation length that is a measure of the extent of the aggregated network.

The unfolding of protein on applying pressure or in presence of urea is believed to be opening of the globular protein structure into a random coil Gaussian conformation of the unfolded polypeptide chain [4,32]. In this case, scattering cross section is given as [54]

$$
\frac{d \Sigma}{d \Omega}(Q)=I_{0}\left[Q^{2} R_{g}^{2}-1+\exp \left(-Q^{2} R_{g}^{2}\right)\right] /\left(Q R_{g}\right)^{4}
$$

where $R_{g}$ is the radius of gyration of the unfolded protein polypeptide chain.

The denaturation of protein in presence of surfactant has been treated using the necklace model of protein-surfactant complexes that assumes micellelike clusters of surfactant randomly distributed along the unfolded polypeptide chain. The cross section for such a system can be written as [33]

$$
\frac{d \Sigma_{s}}{d \Omega}(Q)=\frac{N_{1}^{2}}{N_{p} N}\left(b_{m}-V_{m} \rho_{s}\right)^{2} P_{m}(Q) S_{f}(Q)+B,
$$

where $N_{1}$ is the number density of the total surfactant molecules in solution. $V_{m}$ the volume of the micelle and $N$ the number of such micelles attached to a polypeptide chain. $b_{m}$ represents the scattering length of the surfactant molecule. $P_{m}(Q)$ denotes the normalized intraparticle structure factor of a single micellelike cluster, which for a spherical micelles is the same as given by Eq. (7). $S_{f}(Q)$ for such a system is expressed as in Eq. (8).

\section{B. Dynamic light scattering}

The signal generated by the light scattering from diffusing particles can be analyzed by its intensity autocorrelation function $G^{I}(\tau)[55]$

$$
G^{I}(\tau)=\langle I(t) I(t+\tau)\rangle
$$

where $I(t)$ is the scattered light intensity at time $t$ and $I(t$ $+\tau)$ the scattered light intensity at time $t$ plus a lag time $\tau$. The normalized intensity autocorrelation function $g^{I}(\tau)$ is

$$
g^{I}(\tau)=\frac{G^{I}(\tau)}{\langle I(t)\rangle^{2}}
$$

The electric field autocorrelation function $g^{E}(\tau)$ is related to the normalized intensity autocorrelation function by the Siegert relation

$$
g^{I}(\tau)=1+C\left[g^{E}(\tau)\right]^{2},
$$

where $C$ is an experimental parameter which mainly depends on the detection optics and alignment. For a monodisperse system of particles, $g^{E}(\tau)$ follows a simple exponential decay with decay constant $\gamma$

$$
g^{E}(\tau)=\exp [-\gamma \tau] .
$$

The average decay rate $(\gamma)$ of $g^{E}(\tau)$ has been estimated using a monomodal fit. The apparent diffusion coefficient $\left(D_{a}\right)$ is obtained from the relation $\gamma=D_{a} Q^{2}$ and the corresponding effective hydrodynamic size $\left(R_{H}\right)$ calculated using Stokes-Einstein relationship as given by

$$
R_{H}=\frac{k_{B} T}{6 \pi \eta D_{a}}
$$

where $k_{B}$ is the Boltzmann constant, $T$ is the temperature and $\eta$ is the viscosity of the solvent. The parameters in the analysis were optimized by means of nonlinear least square fitting program and the errors (standard deviations) on the parameters wherever possible were calculated by standard methods used [56]

\section{RESULTS AND DISCUSSION}

SANS data on temperature effect for $1 \mathrm{wt} \% \mathrm{BSA}$ are shown in Fig. 1. Based on the changes in the scattering profile the data have been grouped in two different sets. The first set consists of SANS data for temperature variation from 

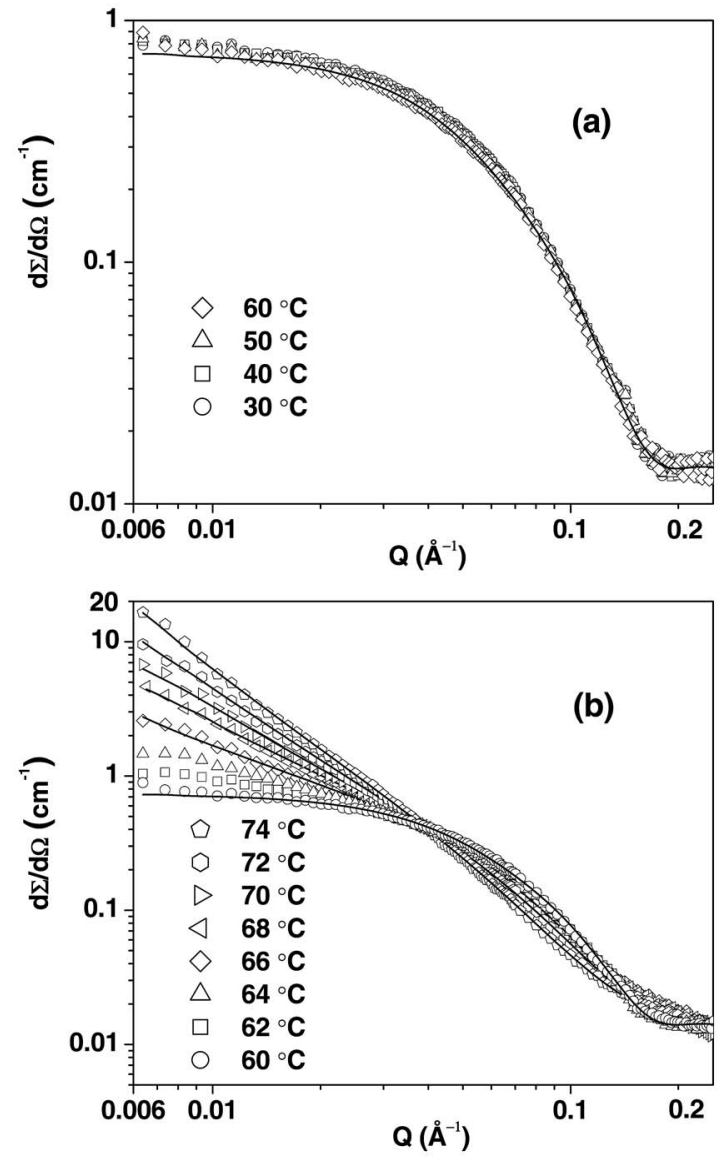

FIG. 1. SANS data for 1 wt. $\%$ BSA at temperatures (a) 30 to $60{ }^{\circ} \mathrm{C}$ and (b) 60 to $74{ }^{\circ} \mathrm{C}$.

ambient to $60^{\circ} \mathrm{C}$ [Fig. 1(a)], which does not show any change in the scattering profile. This suggests that a temperature as high as $60^{\circ} \mathrm{C}$ has no prominent effect on the structure of protein macromolecule and that the protein structure remains stable up to this temperature. This result is in agreement with a previous study using Fourier transform infrared spectroscopy on temperature-induced denaturation [57], which showed no loss of the native structure up to a temperature of around $60{ }^{\circ} \mathrm{C}$. SANS data for the native structure of a protein has been fitted using Eq. (2). It is found that the protein macromolecules are prolate ellipsoidal in shape with semimajor and semiminor axes $a=70.2 \pm 5.1$ and $b=c$ $=22.2 \pm 0.8 \AA$, respectively, which are similar to values reported earlier [34]. Figure 1(b) shows SANS data for temperatures higher than $60{ }^{\circ} \mathrm{C}$. In this case there is build-up of scattering cross section in the low- $Q$ region with increase in temperature. It is believed that protein denaturation at higher temperatures occurs due to exposure of hydrophobic groups of protein to water $[12,13]$. To hide these entropically unfavorable exposed hydrophobic patches, aggregation takes place amongst protein macromolecules. The build-up of the cross section in Fig. 1(b) in the low- $Q$ region is an indication of such aggregation at higher temperatures. The increase in scattering cross section suggests that the aggregation is enhanced with increasing temperature, which finally leads to gelation. We have observed that the present system shows the formation of gel at $65{ }^{\circ} \mathrm{C}$. At this temperature, the visual
TABLE I. Fitted parameters of SANS analysis for gel structure of 1 wt. \% BSA at higher temperatures. This protein maintains a native structure up to $60{ }^{\circ} \mathrm{C}$ and has a prolate ellipsoidal shape with the semimajor axis $a=71.0 \AA$ and semiminor $b=c=22.0 \AA$.

\begin{tabular}{lcc}
\hline \hline $\begin{array}{l}\text { Temperature } \\
\left({ }^{\circ} \mathrm{C}\right)\end{array}$ & $\begin{array}{l}\text { Radius } \\
R_{p}(\AA)\end{array}$ & $\begin{array}{c}\text { Fractal dimension } \\
D\end{array}$ \\
\hline 66 & $24.1 \pm 0.9$ & $1.12 \pm 0.03$ \\
68 & $25.5 \pm 1.0$ & $1.46 \pm 0.04$ \\
70 & $25.5 \pm 1.0$ & $1.66 \pm 0.06$ \\
72 & $25.5 \pm 1.0$ & $1.88 \pm 0.10$ \\
74 & $25.5 \pm 1.0$ & $2.01 \pm 0.12$ \\
\hline \hline
\end{tabular}

turbidity of the protein solution increases dramatically, and SANS data beyond this gelation temperature shows a linear region of scattering cross section on log-log scale for values of $Q<0.07 \AA^{-1}$ [Fig. 1(b)]. This represents the fractal structure of a gel which consists of a network kind of arrangement of protein aggregates in the system. The slope of the scattering data gives the value of the fractal dimension $D$ of the network. The cutoffs of the linear range of the data at low and high $Q$ values are, respectively, related to the extent of the aggregated network and the size of the building block of the network. The low $Q$ cutoff is not observed in Fig. 1(b), where the lowest $Q$ value is $Q_{\min }=0.006 \AA^{-1}$. This means that the aggregated network has a size $\xi$ larger than $2 \pi / Q_{\min }$ (i.e., $900 \AA$ ). The fitted parameters using Eq. (6) are given in Table I. It is found that the fractal dimension increases with increasing temperature. This is understood to be due to the increase in branching between the aggregated protein molecules with increasing temperature. The size of the building block of the aggregated network $\left(R_{p} \sim 25 \pm 1 \AA\right)$ has been found to be significantly smaller than the equivalent spherical radius $\left[\left(a b^{2}\right)^{1 / 3}=32.5 \pm 3.5 \AA\right]$ of the native structure of the protein. This can be possible due to protein undergoing into different structural changes while aggregation occurs [12]. For example, a change of inner interactions of the protein due to temperature increase could give a different packing of the groups composing the protein. It could also be possible that temperature denaturation breaks the protein into smaller hydrophobic patches.

DLS data for $1 \mathrm{wt}$ \% protein solution at increasing temperatures are shown in Fig. 2. DLS measures the timedependent fluctuations in the intensity of scattered light [55]. These fluctuations happen as a result of the Brownian motion. Small particles diffuse rapidly and yield fast fluctuations, whereas large particles and aggregates generate relatively slow fluctuations. The rate of the fluctuations is determined through the autocorrelation analysis technique. The calculated autocorrelation function enables the determination of the diffusion coefficient [Eq. (14)], which then can be converted to a size using the Stokes-Einstein relationship [Eq. (15)]. The DLS data up to $60{ }^{\circ} \mathrm{C}$ [Fig. 2(a)] shows faster decays of the intensity autocorrelation function with increasing temperature. The correlation functions suggest that the diffusion coefficient increases with increasing temperature. On the other hand, the viscosity of the medium is known to decrease with increase in temperature [58]. When 

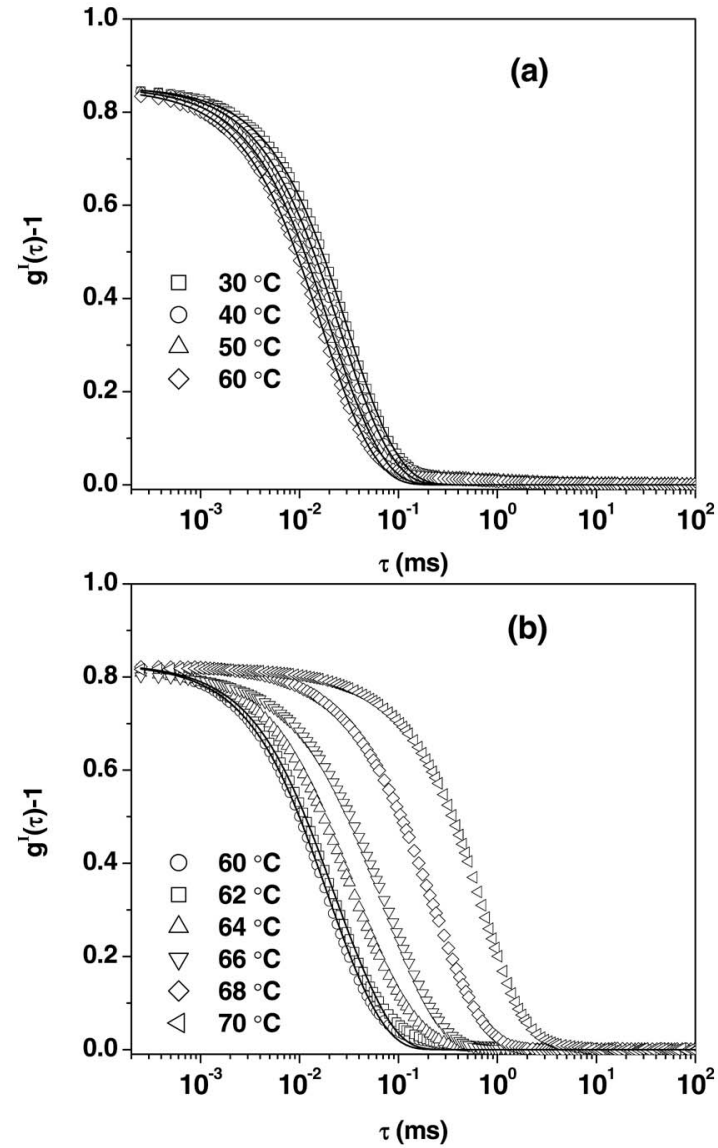

FIG. 2. DLS data for 1 wt. $\%$ BSA at temperatures (a) 30 to $60{ }^{\circ} \mathrm{C}$ and (b) 30 to $70{ }^{\circ} \mathrm{C}$.

$D_{a}$ is corrected for the compensating effect of $\eta$, it is found that the hydrodynamic size as obtained by Stokes-Einsten equation (15) remains the same on increasing temperature (see Table II). These results on protein solution up to $60{ }^{\circ} \mathrm{C}$ are thus consistent with those obtained using SANS. On increasing the temperature above $60{ }^{\circ} \mathrm{C}$ [Fig. 2(b)], there is a strong decrease in the decay rate of the intensity autocorrelation function. The progressive slow diffusion in the system with increase in temperature is consistent with the occurrence of gelation followed by aggregation as observed using SANS.

Figure 3(a) shows the DLS data for pressure effect on $1 \mathrm{wt} . \%$ protein solution. It is observed that the intensity autocorrelation function does not show any significant change

TABLE II. Fitted parameters of DLS analysis for 1 wt. \% BSA as a function of increasing temperature.

\begin{tabular}{lccc}
\hline \hline $\begin{array}{l}\text { Temperature } \\
\left({ }^{\circ} \mathrm{C}\right)\end{array}$ & $\begin{array}{c}\text { Diffusion } \\
\text { coefficient } \\
D_{a}\left(10^{-8} \mathrm{~cm}^{2} / \mathrm{s}\right)\end{array}$ & $\begin{array}{c}\text { Solvent } \\
\text { viscosity } \\
\eta(\mathrm{mPas})\end{array}$ & $\begin{array}{c}\text { Hydrodynamic } \\
\text { radius } \\
R_{H}(\AA)\end{array}$ \\
\hline 30 & 64.30 & 1.03 & 33.5 \\
40 & 75.13 & 0.86 & 33.8 \\
50 & 83.56 & 0.72 & 34.1 \\
60 & 96.30 & 0.61 & 34.7 \\
\hline \hline
\end{tabular}
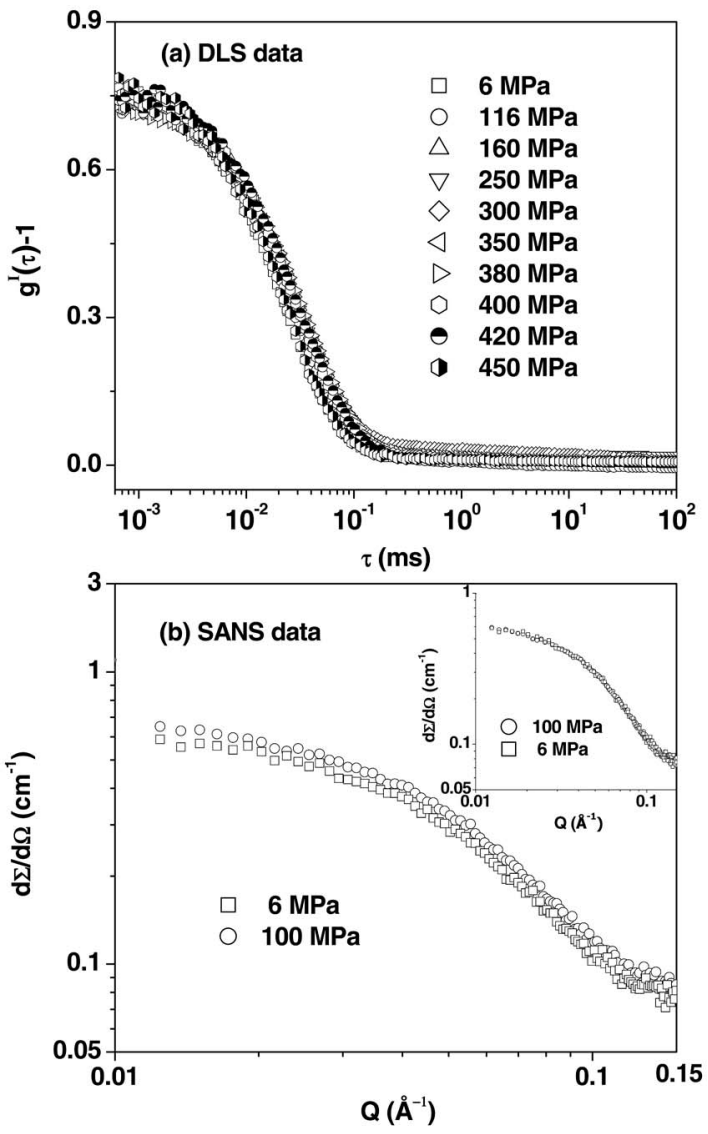

FIG. 3. DLS and SANS data for 1 wt. $\%$ BSA as a function of pressure.

up to a pressure of $450 \mathrm{MPa}$. Pressure-induced denaturation is believed to be due to water penetration inside the core of the protein which decreases the hydrophobic interactions among nonpolar groups of the protein molecule $[14,15]$. It has been found for small globular proteins such as staphylococcal nuclease (SN) [59] that pressure beyond $200 \mathrm{MPa}$ can unfold these proteins. We do not observe any protein unfolding by applying pressure up to $450 \mathrm{MPa}$, it may be perhaps due to the fact that BSA $(66.4 \mathrm{KDa})$ is much larger protein than $[\mathrm{SN}(16.8 \mathrm{KDa})]$ for which pressure induced unfolding has been observed in this pressure range. Larger size of the protein means higher hydrophobic interactions between the nonpolar groups inside the protein [60] and therefore larger pressure is needed to weaken these interactions. SANS data on 1 wt. $\%$ protein solution at $100 \mathrm{MPa}$ are shown in Fig. 3(b). It is found that there is an increase in scattering cross section on applying the pressure. However, the functionality of the scattering profile remains same (see the inset of the figure). The increase in the scattering cross section can be understood in terms of increase in number density of the protein macromolecules and the scattering length density of the solvent (i.e., contrast factor) as the system gets compressed on applying pressure [61]. We could not perform the SANS data at higher pressures as the measurements take longer time than that in DLS for which it was difficult to maintain the pressure.

SANS and DLS data for 1 wt. $\%$ BSA in presence of a varying concentration of urea are shown in Fig. 4. SANS 

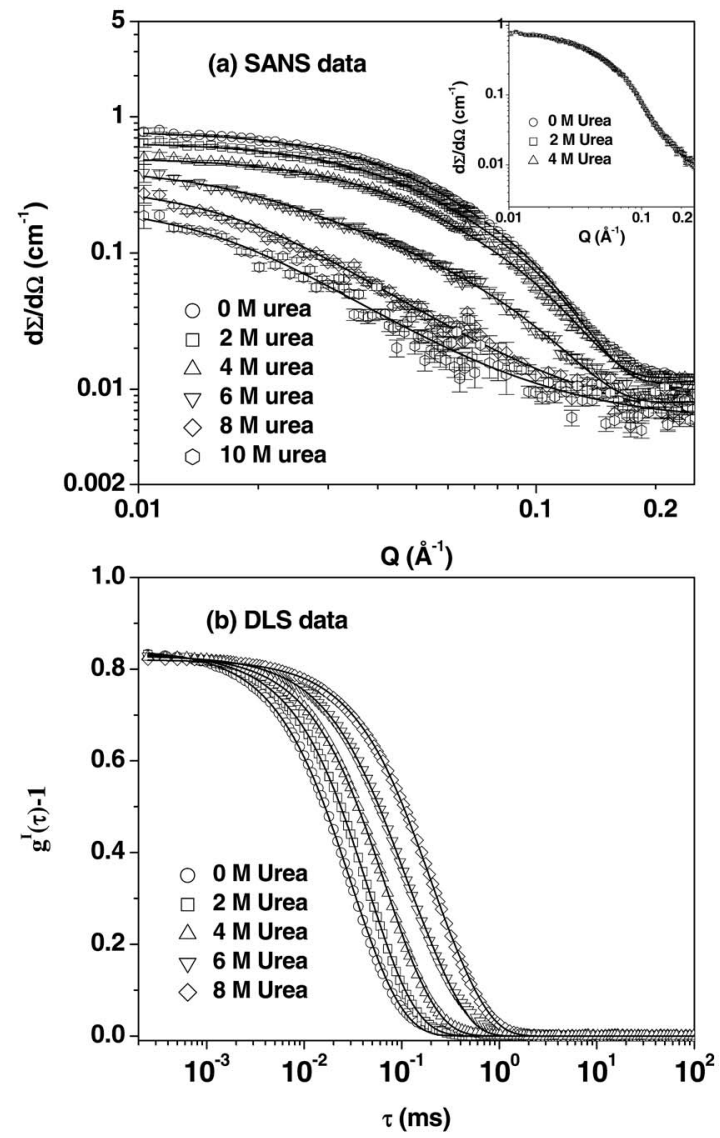

FIG. 4. SANS and DLS data for 1 wt. $\%$ BSA in presence of varying urea concentration.

data [Fig. 4(a)] show decrease in scattering cross section with increasing urea concentration. It is observed that up to $4 M$ concentration of urea, there is a continuous decrease in the scattering cross section, however, the functionality of the scattering pattern does not change. The inset of the Fig. 4(a) shows the scaling of the data suggesting the same functionality of the scattering profiles for urea concentrations in the range $0 M$ to $4 M$. The decrease in scattering cross section can be explained in terms of decrease in contrast $\left(\rho_{p}-\rho_{s}\right)^{2}$ as the scattering length density of deuterated solvent $\left(\rho_{s}\right)$ decreases on addition of hydrogenous urea to protein solution. There is a change in the functionality of the scattering profile beyond $4 M$ urea and it is interpreted in terms of unfolding of the protein. It is believed that the solvation of hydrophobic portions of the protein at high urea concentrations leads to the unfolding of a protein. The unfolded protein is fitted as random Gaussian coil using Eq. (8). It is found that the radius of gyration $\left(R_{g}\right)$ of the unfolded protein increases with increasing urea concentration. The value of $R_{g}$ increases from $55.0 \pm 2.9$ to $93.5 \pm 6.4 \AA$ as the urea concentration is increased from $6 M$ to $10 M$ (Table III). DLS data on addition of urea to protein solution are shown in Fig. 4(b). There is a decrease in the average decay constant indicating a slowing down of the diffusion of the protein macromolecules on increasing urea concentration. The calculated hydrodynamic size from the DLS data is shown in Table IV. This analysis takes account of the increase in the solvent viscosity on ad-
TABLE III. Fitted parameters of SANS analysis for 1 wt. \% BSA in presence of varying urea concentrations. The protein has a prolate ellipsoidal shape up to $4 M$ urea beyond this concentration it unfolds into a random coil conformation.

\begin{tabular}{lccc}
\hline \hline $\begin{array}{l}\text { [Urea] } \\
(M)\end{array}$ & $\begin{array}{c}\text { Semiminor axis } \\
a(\AA)\end{array}$ & $\begin{array}{c}\text { Semimajor axis } \\
b=c(\AA)\end{array}$ & $R_{g}(\AA)$ \\
\hline 0 & $22.2 \pm 0.8$ & $71.0 \pm 5.1$ & \\
2 & $22.2 \pm 0.8$ & $71.0 \pm 5.1$ & \\
4 & $22.2 \pm 0.8$ & $71.0 \pm 5.1$ & \\
6 & & & $55.0 \pm 2.9$ \\
8 & & & $84.0 \pm 4.1$ \\
10 & & & $93.5 \pm 6.4$ \\
\hline \hline
\end{tabular}

dition of urea [62]. The hydrodynamic size remains similar up to $4 M$ urea concentration and increases beyond this concentration as the protein unfolds, which is consistent with the SANS results (Table III).

The reversibility of protein unfolding in presence of urea has been examined using dilution method and the data are shown in Fig. 5. In this method, the urea concentration in the protein solution is reduced through the dilution of the sample. This method will also reduce the protein concentration and therefore it will only work if the protein unfolding or folding is independent of protein concentration. Figure 5(a) shows the SANS data for two protein concentrations (1 and $0.5 \mathrm{wt} . \%$ ) with and without $8 M$ urea. It is found that the data for the two concentrations can be scaled by a factor 2 (protein concentration ratio), which confirms that the folded protein structure (without urea) and the unfolded protein structure ( $8 M$ urea) is independent of protein concentrations of present interest. Figure 5(b) shows the SANS data for 0.5 wt. $\%$ protein in presence of 4 and $8 M$ urea concentrations corresponding to folded and unfolded structures, respectively. Figure 5(b) also shows SANS data for $0.5 \mathrm{wt} \%$ BSA with $4 M$ urea, which is obtained by diluting to half the concentration of 1 wt. \% BSA with $8 M$ urea. It is interesting to observe that while the functionality of $0.5 \mathrm{wt}$. \% BSA with $8 M$ urea and 1 wt. $\%$ BSA with $8 M$ urea are similar [Fig. $5(\mathrm{a})]$, the data of 0.5 wt. $\%$ BSA with $4 M$ urea and the diluted 1 wt. $\%$ BSA with $8 M$ urea to 0.5 wt. $\%$ BSA with $4 M$ urea are also similar. This suggests that the unfolded protein in 1 wt. \% BSA with $8 M$ urea gets back to the folded structure on reducing the urea concentration by the dilution

TABLE IV. Fitted parameters of DLS analysis for $1 \mathrm{wt}$ \% BSA in presence of varying urea concentrations.

\begin{tabular}{lccc}
\hline \hline $\begin{array}{l}\text { [Urea] } \\
(M)\end{array}$ & $\begin{array}{c}\text { Diffusion } \\
\text { coefficient } \\
D_{a}\left(10^{-8} \mathrm{~cm}^{2} / \mathrm{s}\right)\end{array}$ & $\begin{array}{c}\text { Solvent } \\
\text { viscosity } \\
\eta(\mathrm{mPa} \mathrm{s})\end{array}$ & $\begin{array}{c}\text { Hydrodynamic } \\
\text { radius } \\
R_{H}(\AA)\end{array}$ \\
\hline 0 & 64.3 & 1.03 & 33.5 \\
2 & 63.6 & 1.12 & 34.1 \\
4 & 61.2 & 1.25 & 34.5 \\
6 & 43.6 & 1.44 & 68.5 \\
8 & 18.3 & 1.71 & 136.5 \\
\hline \hline
\end{tabular}



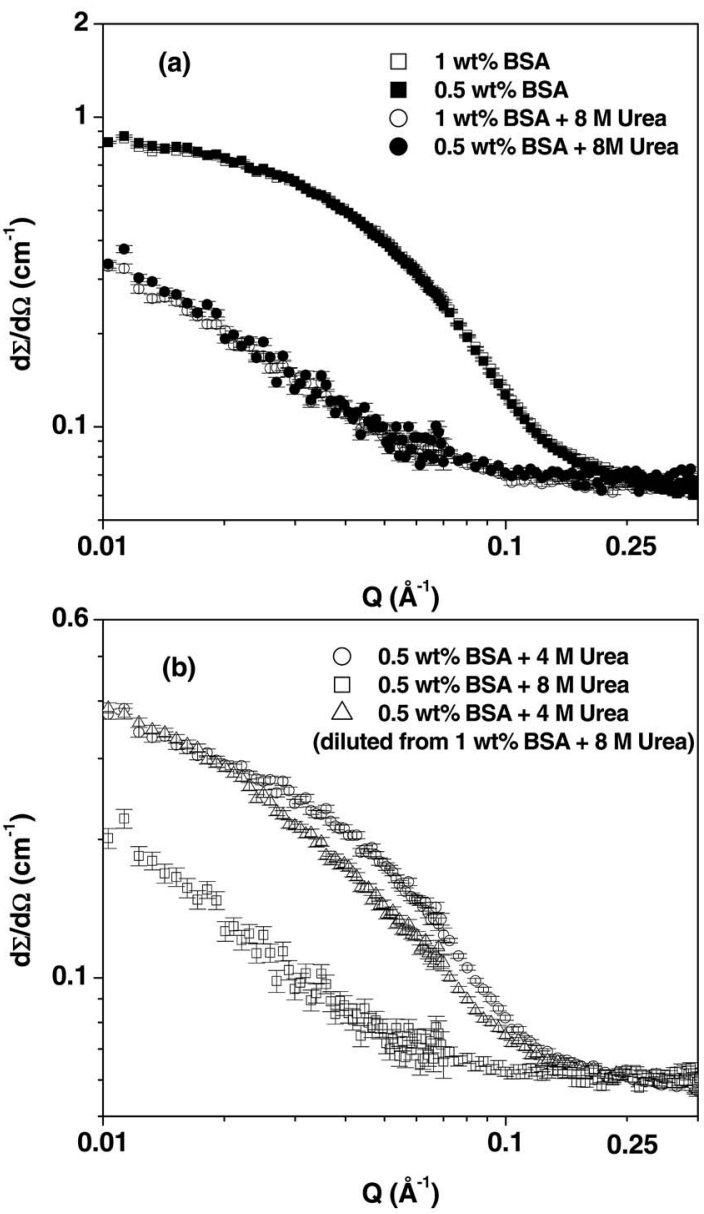

FIG. 5. SANS data for (a) 1 and 0.5 wt. $\%$ BSA in pure solutions and with $8 M$ urea where the data of $0.5 \mathrm{wt} \%$ BSA are scaled by a factor of 2 for concentration correction, (b) 0.5 wt. \% BSA in presence of 4 and $8 M$ urea along with 0.5 wt. $\%$ BSA with $4 M$ urea diluted from 1 wt. \% BSA in presence of $8 M$ urea.

method. The small difference between 0.5 wt. \% BSA with $4 M$ urea and the one diluted from $8 M$ to $4 M$ urea may be due to that protein unfolding is not completely reversible. The shape of this folded protein structure is also found to prolate ellipsoidal having the semimajor and semiminor axes as $a=74.5 \pm 5.3$ and $b=c=23.1 \pm 0.9 \AA$, respectively. The axes of the folded and refolded proteins are the same in the limit of the experimental errors.

We have recently reported SANS and DLS studies on $1 \mathrm{wt}$ \% BSA with the addition of surfactant sodium dodecyl sulfate (SDS) over a wide concentration range of surfactant [34]. On the basis of the necklace model of the proteinsurfactant complex which considers micellelike clusters of the surfactant formed along the unfolded polypeptide chain of the protein, a fractal structure is used to represent the complex [Eq. (10)]. It has been found that the fractal dimension decreases and the overall size of the complex increases with increasing surfactant concentration. The size of the micellelike clusters does not change while the number of such micellelike clusters in the protein-surfactant complex increases with the surfactant concentration. The calculated aggregation number of micellelike clusters in the complex is
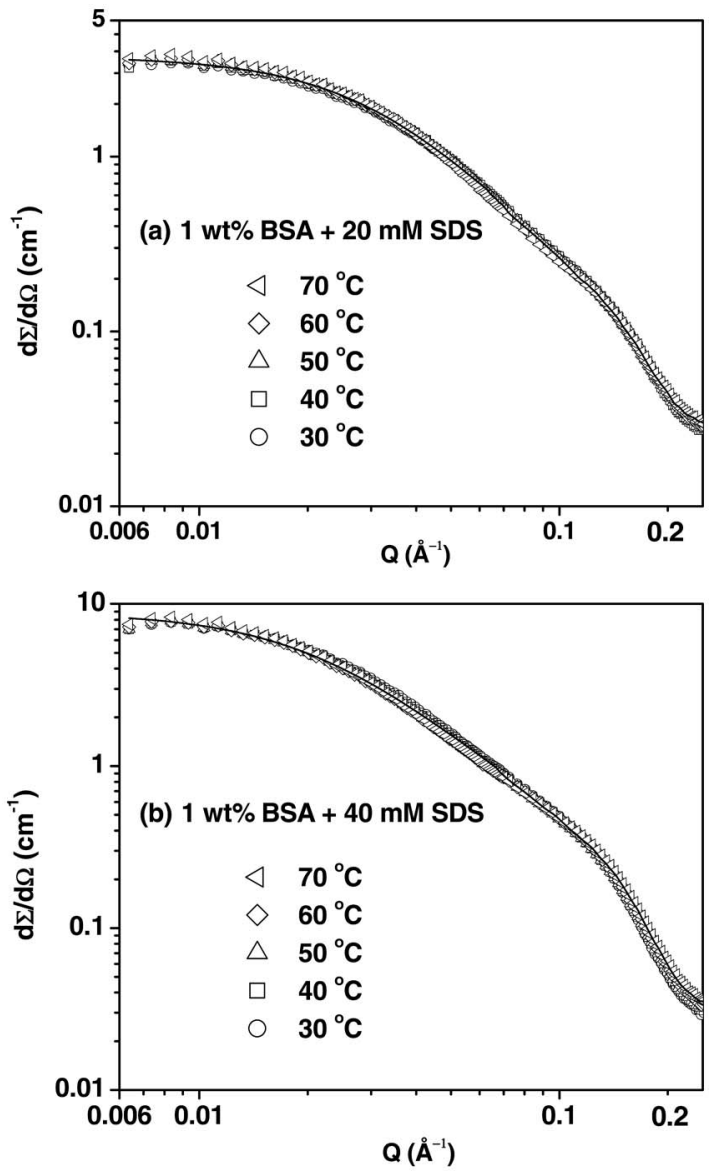

FIG. 6. SANS data for $1 \mathrm{wt} \%$ BSA in presence of 20 and $40 \mathrm{~m} M$ SDS as a function of temperature.

found to be much smaller than one would find in pure surfactant solution for the similar size of micelles [63]. This indicates the participation of the hydrophobic portions of the unfolded protein chain in the micellar formation [64]. The temperature effect has now been studied to examine the stability of these protein-surfactant complexes.

In pure protein solution there is aggregation followed by gelation at higher temperatures (Table I). Temperature induced gelation occurs due to hydrophobic groups in protein, which remain buried inside the protein at ambient temperature and become exposed to water at higher temperatures. The formation of a protein-surfactant complex can be used in this regard to control the temperature induced protein gelation as the micellelike clusters formed in the complex hide the hydrophobic groups of the proteins and prevent the aggregation amongst themselves. SANS data of temperature effect on protein-surfactant complexes of $1 \mathrm{wt}$. \% BSA with 20 and $40 \mathrm{~m} M$ SDS are shown in Fig. 6. The fitted parameters in these systems using Eq. (10) are given in Table V. It is observed that unlike the pure protein solution, which shows gelation at higher temperatures (Table I), there is almost no effect of temperature on the protein-surfactant complex. These results suggest that the presence of surfactant in a protein-surfactant complex stabilizes the complex against any aggregation. Although the SANS data have been measured up to $70{ }^{\circ} \mathrm{C}$, however, we have observed visually that 
TABLE V. Fitted parameters of SANS analysis for $1 \mathrm{wt}$ \% BSA in presence of $\mathrm{C} \mathrm{m} M$ SDS as a function of varying temperature.

\begin{tabular}{|c|c|c|c|c|c|}
\hline \multicolumn{6}{|c|}{ (a) $1 \mathrm{wt} . \% \mathrm{BSA}$ in presence of $20 \mathrm{M}$ SDS } \\
\hline $\begin{array}{l}\text { Temperature } \\
\left({ }^{\circ} \mathrm{C}\right)\end{array}$ & $\begin{array}{c}\text { Fractal } \\
\text { dimension } \\
D\end{array}$ & $\begin{array}{l}\text { Correlation } \\
\text { length } \xi \\
(\AA)\end{array}$ & $\begin{array}{c}\text { Micelle } \\
\text { Radius } R_{m} \\
(\AA)\end{array}$ & $\begin{array}{c}\text { Number of } \\
\text { micelles } \\
N\end{array}$ & $\begin{array}{c}\text { Aggregation } \\
\text { Number } \\
n\end{array}$ \\
\hline 30 & $2.27 \pm 0.15$ & $38.0 \pm 1.9$ & $18.0 \pm 0.6$ & 2 & 51 \\
\hline 40 & $2.32 \pm 0.15$ & $37.8 \pm 1.9$ & $18.0 \pm 0.6$ & 2 & 51 \\
\hline 50 & $2.29 \pm 0.15$ & $38.1 \pm 1.9$ & $18.0 \pm 0.6$ & 2 & 51 \\
\hline 60 & $2.34 \pm 0.15$ & $37.6 \pm 1.9$ & $18.0 \pm 0.6$ & 2 & 51 \\
\hline 70 & $2.29 \pm 0.15$ & $38.2 \pm 1.9$ & $18.0 \pm 0.6$ & 2 & 51 \\
\hline \multicolumn{6}{|c|}{ (b) $1 \mathrm{wt} \% \mathrm{BSA}$ in presence of $40 \mathrm{~m} M$ SDS } \\
\hline 30 & $2.05 \pm 0.13$ & $54.3 \pm 3.8$ & $18.0 \pm 0.6$ & 4 & 50 \\
\hline 40 & $2.02 \pm 0.13$ & $54.8 \pm 3.8$ & $18.0 \pm 0.6$ & 4 & 50 \\
\hline 50 & $1.99 \pm 0.13$ & $56.9 \pm 3.8$ & $18.0 \pm 0.6$ & 4 & 50 \\
\hline 60 & $2.01 \pm 0.13$ & $56.3 \pm 3.8$ & $18.0 \pm 0.6$ & 4 & 50 \\
\hline 70 & $2.03 \pm 0.13$ & $55.1 \pm 3.8$ & $18.0 \pm 0.6$ & 4 & 50 \\
\hline
\end{tabular}

no gelation occurs in these systems even temperature up to $95^{\circ} \mathrm{C}$.

\section{CONCLUSIONS}

The denaturation of BSA proteins has been studied using SANS and DLS as a function of the different variables temperature, pressure, urea and surfactant. It is found that the protein maintains its native structure up to a temperature as high as $60{ }^{\circ} \mathrm{C}$. Beyond this temperature there is aggregation amongst protein macromolecules which leads to gelation. The protein gel is characterized by a fractal structure and the fractal dimension of this structure increases with rise in temperature due to enhanced branching of the aggregates. There is no protein denaturation observed for BSA on application of pressure up to $450 \mathrm{MPa}$. The addition of urea leads to denaturation of the protein only at concentrations higher than
$4 M$. For urea concentrations above $4 M$, the protein unfolds and acquires a random coil Gaussian conformation, whose radius of gyration increases with increasing urea concentration. The urea-induced protein unfolding is demonstrated to be reversible. The denaturation of protein in presence of surfactant is found to be caused by the formation of micellelike clusters along the unfolded protein polypeptide chain. The temperature induced gelation in pure protein solution is found to be suppressed on addition of surfactant.

\section{ACKNOWLEDGMENTS}

The authors wish to thank Dr. F. Pfeiffer and M. Dierolf for their kind help during the DLS experiments. This work is based on SANS experiments performed at the Swiss spallation neutron source SINQ, Paul Scherrer Institut, Villigen, Switzerland.
[1] C. Tanford, Adv. Protein Chem. 24, 1 (1970).

[2] C. M. Dobson, Nature (London) 426, 884 (2003).

[3] D. Paschek and A. E. Garcia, Phys. Rev. Lett. 93, 238105 (2004).

[4] R. S. Tu and V. Breedveld, Phys. Rev. E 72, 041914 (2005).

[5] A. Valstar, M. Almgren, W. Brown, and M. Vasilescu, Langmuir 16, 922 (2000).

[6] A. H. Clark, G. M. Kavanagh, and S. B. Ross-Murphy, Food Hydrocolloids 15, 383 (2001).

[7] T. Hagiwara, H. Kumagai, and K. Nakamura, Food Hydrocolloids 12, 29 (1998).

[8] R. S. Meyer, K. L. Cooper, D. Knorr, and H. L. M. Lelieveld, Food Technol. Aust. 54, 67 (2000).

[9] E. D. Goddard and K. P. Ananthapadmanabhan, Interactions of Surfactants with Polymers and Proteins (CRC, London, 1993).
[10] C. Sun, J. Yang, X. Wu, X. Huang, F. Wang, and S. Liu, Biophys. J. 88, 3518 (2005).

[11] C. C. Chang, Y. C. Su, M. S. Cheng, and L. S. Kan, Phys. Rev. E 66, 021903 (2002).

[12] W. S. Gosal and S. B. Ross-Murphy, Curr. Opin. Colloid Interface Sci. 5, 188 (2000).

[13] S. B. Ross-Murphy, Phys. Chem. Chem. Phys. 102, 1534 (1998).

[14] G. Panik, R. Malessa, and R. Winter, Biochemistry 38, 6512 (1999).

[15] G. Panik, R. Malessa, R. Winter, G. Rapp, K. J. Frye, and C. A. Royer, J. Mol. Biol. 275, 389 (1998).

[16] M. Ikeguchi, S. Nakamura, and K. Shimizu, J. Am. Chem. Soc. 123, 677 (2001).

[17] R. D. Mountain and D. Thirumalai, J. Am. Chem. Soc. 125, 
1950 (2003).

[18] D. Tobi, R. Elber, and D. Thirumalai, Biopolymers 68, 359 (2003).

[19] M. Lee and N. F. A. Van der Vegt, J. Am. Chem. Soc. 128, 4948 (2006).

[20] A. Wallqvist, D. G. Covell, and D. J. Thirumalai, J. Am. Chem. Soc. 120, 427 (1998).

[21] F. Vanzi, B. Madan, and K. Sharp, J. Am. Chem. Soc. 120, 10748 (1998).

[22] S. Shimizu and S. H. Chan, Proteins 49, 560 (2002).

[23] B. J. Bennion and V. Dagget, Proc. Natl. Acad. Sci. U.S.A. 125, 1950 (2003).

[24] M. Vasilescu, D. Angelescu, M. Almgren, and A. Valstar, Langmuir 15, 2635 (1999).

[25] E. L. Gelamo, R. Itri, A. Alonso, J. V. da Silva, and M. Tabak, J. Colloid Interface Sci. 277, 471 (2004).

[26] M. N. Jones, H. A. Skinner, and E. Tipping, Biochem. J. 147, 229 (1975).

[27] J. C. Gimel and W. Brown, J. Chem. Phys. 104, 8112 (1996).

[28] E. Seth and V. K. Aswal, J. Macromol. Sci., Phys. 42, 85 (2003).

[29] S. Shinagawa, M. Sato, K. Kameyama, and T. Takagi, Langmuir 10, 1690 (1994)

[30] J. Gimel, D. Durand, and T. Nicolai, Macromolecules 27, 583 (1994).

[31] S. Ikeda, E. A. Foegeding, and T. Hagiwara, Langmuir 15, 8584 (1999).

[32] Y. Harano and M. Kinoshita, J. Phys.: Condens. Matter 18, L107 (2006).

[33] S. H. Chen and J. Teixeira, Phys. Rev. Lett. 57, 2583 (1986).

[34] S. Chodankar, V. K. Aswal, J. Kohlbrecher, R. Vavrin, and A. G. Wagh, J. Phys.: Condens. Matter 19, 326102 (2007).

[35] A. K. Morén and A. Khan, Langmuir 14, 6818 (1998).

[36] M. L. Anson and A. E. Mirsky, J. Gen. Physiol. 15, 341 (1931).

[37] S. Deep and J. C. Ahluwalia, Phys. Chem. Chem. Phys. 3, 4583 (2001).

[38] Q. Xu and T. A. Keiderling, Protein Sci. 13, 2949 (2004).

[39] A. Stenstam, D. Topgaard, and H. Wennerström, J. Phys. Chem. B 107, 7987 (2003).

[40] C. C. McDonald and W. D. Phillips, J. Am. Chem. Soc. 89,
6332 (1967).

[41] J. C. Gimel and W. Brown, J. Chem. Phys. 104, 8112 (1996).

[42] X. H. Guo, N. M. Zhao, S. H. Chen, and J. Teixeira, Biopolymers 29, 335 (1990).

[43] D. I. Svergun and M. H. J. Koch, Rep. Prog. Phys. 66, 1735 (2002).

[44] J. Kohlbrecher and W. Wagner, J. Appl. Crystallogr. 33, 804 (2000).

[45] U. Keiderling, Appl. Phys. A 74, S1455 (2002).

[46] P. Schiebener and J. Straub, J. Phys. Chem. Ref. Data 19, 677 (1996).

[47] J. R. Warren and J. A. Gordon, J. Phys. Chem. 70, 297 (1966).

[48] J. Kohlbrecher, A. Bollhalder, R. Vavrin, G. Meier, and J. Buitenhuis, Rev. Sci. Instrum. 78, 125101 (2007).

[49] J. B. Hayter and J. Penfold, Colloid Polym. Sci. 261, 1022 (1983).

[50] S. Chodankar and V. K. Aswal, Phys. Rev. E 72, 041931 (2005).

[51] J. S. Pedersen, Adv. Colloid Interface Sci. 70, 171 (1997).

[52] P. W. Schmidt, J. Appl. Crystallogr. 24, 414 (1991).

[53] J. Teixeira, J. Appl. Crystallogr. 21, 781 (1988).

[54] P. Debye, J. Phys. Colloid Chem. 51, 18 (1947).

[55] R. Pecora, Dynamic Light Scattering (Plenum, New York, 1985).

[56] P. R. Bevington, Data Reduction and Error Analysis for Physical Sciences (McGraw-Hill, New York, 1969).

[57] K. Murayama and M. Timoda, Biochemistry 43, 11526 (2004).

[58] http://physchem.kfunigraz.ac.at/sm/Service/Water/ D2Ovisc.htm\#lit1

[59] A. Paliwal, D. Asthagiri, D. P. Bossev, and M. E. Paulaitis, Biophys. J. 87, 3479 (2004).

[60] C. Tanford, The Hydrophobic Effect: Formation of Micelles and Biological Membranes (Wiley, New York, 1980).

[61] M. S. Appavou, G. Gibrat, and M. C. Bellissent-Funel, Biochim. Biophys. Acta 1764, 414 (2006).

[62] K. Kawahara and C. Tanford, J. Biol. Chem. 241, 3228 (1966).

[63] V. K. Aswal and P. S. Goyal, Phys. Rev. E 67, 051401 (2003).

[64] N. J. Turro, K. P. Ananthapadmanabhan, X. G. Lei, and M. Aronson, Langmuir 11, 2525 (1995). 